\title{
More than 10 years survival with sequential therapy in a patient with advanced renal cell carcinoma: a case report
}

\author{
J.L. Yuan ${ }^{1 *}$, F.L. Wang ${ }^{1 *}$, X.M. Yi ${ }^{1 *}$, W.J. Qin ${ }^{1}$, G.J. Wu ${ }^{1}$, Y. Huan ${ }^{2}$, L.J. Yang ${ }^{1}$, G. Zhang ${ }^{1}$, L. Yu ${ }^{1}$, \\ Y.T. Zhang ${ }^{1}$, R.L. Qin ${ }^{1}$ and C.J. Tian ${ }^{1}$
}

${ }^{1}$ Department of Urology, Xijing Hospital, Fourth Military Medical University, Xi'an, Shaanxi, China ${ }^{2}$ Department of Radiology, Xijing Hospital, Fourth Military Medical University, Xi'an, Shaanxi, China

\begin{abstract}
Although radical nephrectomy alone is widely accepted as the standard of care in localized treatment for renal cell carcinoma (RCC), it is not sufficient for the treatment of metastatic RCC (mRCC), which invariably leads to an unfavorable outcome despite the use of multiple therapies. Currently, sequential targeted agents are recommended for the management of mRCC, but the optimal drug sequence is still debated. This case was a 57-year-old man with clear-cell mRCC who received multiple therapies following his first operation in 2003 and has survived for over 10 years with a satisfactory quality of life. The treatments given included several surgeries, immunotherapy, and sequentially administered sorafenib, sunitinib, and everolimus regimens. In the course of mRCC treatment, well-planned surgeries, effective sequential targeted therapies and close follow-up are all of great importance for optimal management and a satisfactory outcome.
\end{abstract}

Key words: Metastatic renal cell carcinoma; Sequential therapy; Targeted anticancer agents; Sorafenib; Sunitinib; Immunotherapy

\section{Introduction}

Renal cell carcinoma (RCC) is the sixth most common malignancy among men and the eighth among women in the USA, and the sixth leading cause of cancer-related deaths $(1,2)$. Currently, radical nephrectomy alone is widely accepted as the standard of care for treatment of localized RCC, but it is not sufficient for the treatment of metastatic RCC (mRCC), which invariably leads to an unfavorable outcome despite the use of multiple therapies. Metastases are found in approximately $30 \%$ of newly diagnosed RCC patients, resulting in a poor 5-year survival rate of less than $10 \%$ (3). However, the availability of several targeted agents [e.g., tyrosine kinase inhibitors such as sorafenib, sunitinib, pazopanib, axitinib, tivozanib, monoclonal antibodies such as bevacizumab (with interferon), and mammalian target of rapamycin (mTOR) inhibitors such as temsirolimus and everolimus] has dramatically changed the treatment of $\operatorname{mRCC}(4,5)$. Extensive use of cytokines such as interferon- $\alpha$ and interleukin- 2 in the past decade has evolved into controlled use of targeted therapies.
$\mathrm{mRCC}$ is one of the most treatment-resistant malignancies. Although targeted agents significantly prolong progression-free survival (PFS) compared with previously used therapies, drug resistance is inevitable. Patients in whom treatment is initially effective almost always experience disease progression (6). As persistent complete remission of $\mathrm{mRCC}$ is rare with current therapeutic modalities, more efficacious treatments with minimal adverse effects are urgently needed. At present, sequential treatment is strongly recommended for the management of $\mathrm{mRCC}$, but the optimal sequenced approach and the prognosis of these patients have not been defined $(7,8)$.

Here, we describe the case of a 57 -year-old male patient with clear-cell mRCC who has survived for more than 10 years with a satisfactory quality of life since undergoing a radical nephrectomy in 2003. During that interval, the patient has benefitted from several advances in the management of $\mathrm{mRCC}$. To the best of our knowledge, this is the first documented case of survival for more than 10 years

Correspondence: Jianlin Yuan: <yuanjianlin317@126.com>

${ }^{*}$ These authors contributed equally to this study.

Received April 29, 2014. Accepted September 22, 2014. First published online October 31, 2014. 
following multiple therapies, including several surgical procedures, cytokine therapy, and sequentially administered targeted agents with long switching intervals.

\section{Case description}

\section{Disease history}

In February 2003, a 57-year-old man (height, $168 \mathrm{~cm}$; body weight, $77 \mathrm{~kg}$ ) presented with painless gross hematuria. Ultrasonography indicated a solid mass in the left kidney. He took Chinese herbal medicines for several months, but the hematuria continued. When he revisited our clinic in October 2003, a computed tomography (CT) scan showed a solid mass of about $8 \times 7 \mathrm{~cm}$ in the lower pole of the left kidney (about $14 \times 11 \mathrm{~cm}$ ) infiltrating the major psoas muscle, with a long embolus in the inferior vena cava (IVC) that reached the entrance of the hepatic vein (Figure 1A). He had a Karnofsky Performance Status (KPS) score of $70 \%$, and Eastern Cooperative Oncology Group (ECOG) performance status of 2 . The patient had a history of chronic gastritis (diagnosed in 1998), hyperlipidemia (diagnosed in 2002), fatty liver disease (diagnosed in 2004), right carotid artery atherosclerosis (diagnosed in 2005), benign prostatic hyperplasia (BPH, diagnosed in 2005), and a left maxillary sinus cyst (diagnosed in 2006), but he is currently taking no medications for these conditions.

In November 2003, the patient underwent radical left

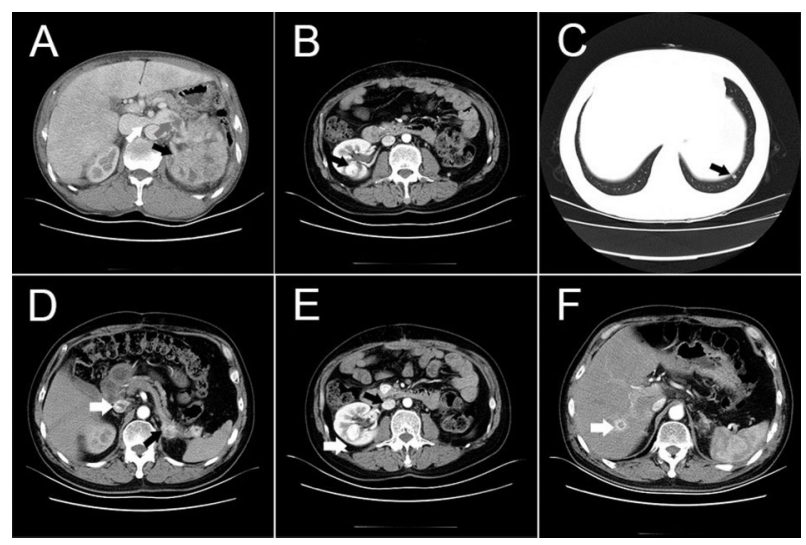

Figure 1. Computed tomography (CT) images of the patient with metastatic renal cell carcinoma at different time points. $A$, CT image before radical nephrectomy of the left kidney (white arrow: embolus in the inferior vena cava (IVC); gray arrow: embolus in the left renal vein; black arrow: the renal tumor site. November 2003). B, CT image before enucleation of two metastases in the right kidney (black arrow: metastasis in the right kidney. June 2006). C, a distinct metastasis in the left lower lung (black arrow, January 2007). D, CT image revealing the embolus in the IVC (white arrow) and the metastasis in the left adrenal gland (black arrow, November 2011). E, a large metastasis in the right kidney (white arrow) and the embolus in the IVC (black arrow, January 2013). F, a round metastatic site was found in the liver (white arrow, January 2013). nephrectomy and removal of the tumor embolus in the IVC The tumor was stage IV according to the 2002 American Joint Committee on Cancer (AJCC) staging system. Postoperative pathological examination confirmed advanced clear-cell carcinoma (pT4N0M0 Fuhrman grade II). Forty days later, CT scans revealed no residue of tumor in the surgical site, but one small (about $0.6 \mathrm{~cm}$ ) nodule was discovered in the left lower lung. Fortunately, the patient was stratified as favorable according to Memorial Sloan-Kettering Cancer Center RCC nomogram. He was started on a combined immunotherapy regimen (interleukin-2 plus interferon), an adjuvant protocol that followed the treatment guidelines for renal cancer recommended by the Chinese Urological Association, and which was adapted for Chinese patients from National Comprehensive Cancer Network (NCCN) practice guidelines (9). The protocol included two cycles of recombinant human interleukin-2 (18 million IU, sc for 5 days in the first week, followed by 3 weeks of 18 million IU for 2 days and 9 million IU for 3 days) and recombinant human interferon- $\alpha 2 \mathrm{~b}$ (9 million $\mathrm{IU}$, im, 3 times per week for 12 weeks). Close follow-up was maintained, including disease history, physical examination, routine blood tests, kidney and liver function tests, and CT imaging every 3 months. In August 2005, a cystic change was noted in the right kidney. Urodynamic tests revealed increased residual urine $(70 \mathrm{~mL})$, and ultrasonography suggested $\mathrm{BPH}$.

In June 2006, two solid masses $(1.0 \times 1.0 \mathrm{~cm}$ and $1.5 \times 1.5 \mathrm{~cm}$ in size) appearing to be metastases were found, in the middle and upper pole of the right kidney (Figure 1B). The small nodule in the left lower lung had not changed significantly. The patient underwent a second surgical procedure via the primary abdominal incision to enucleate the two masses in the right kidney. Intraoperative frozen sections confirmed metastatic clear-cell carcinoma. The patient continued the previous immunotherapy for two cycles after the surgery. Unfortunately, in November 2006, three lung nodules involving both lungs, each smaller than $1 \mathrm{~cm}$, were found.

\section{Sorafenib as first-line targeted therapy}

Sorafenib is recommended as a first-line targeted therapy for $\mathrm{mRCC}$ by the NCCN guidelines, and received marketing approval in China in November 2006. As the patient's mRCC diagnosis satisfied the 2002 AJCC criteria, he began sorafenib therapy, $400 \mathrm{mg}$ twice daily, in January 2007, when the largest of the 3 lung nodules became more apparent, growing to about $1.3 \times 1.5 \mathrm{~cm}$ in size (Figure 1C).

Treatment response was evaluated according to the response evaluation criteria in solid tumors (RECIST) by an expert team that included both a radiologist and a urologist. The disappearance of all known lesions within 1 month was considered a complete response. A greater than $30 \%$ decrease in the total length of longest lesion diameter was considered a partial response, and a greater than $20 \%$ 
increase in the smallest sum as progressive disease. Changes between these limits were defined as stable disease. Adverse events were assessed by National Cancer Institute's Common Terminology Criteria for Adverse Events (CTCAE) 3.0. Grade 1, 2, 3 adverse events that occurred in this patient were described as mild, moderate and severe, respectively.

One week after starting sorafenib therapy, the patient experienced grade 1 hand-foot syndrome, stomatitis, fatigue, and grade 2 diarrhea. These reactions were controlled by symptomatic treatment without the need for dose reduction or therapy interruption. Two months after starting sorafenib therapy, a small embolus was found in the IVC, but the size of the nodules in the lung had decreased. The RECIST evaluation indicated stable disease until January 2011, when CT images revealed a nodule, about $2.9 \times$ $3.0 \mathrm{~cm}$ in size, in the left adrenal gland, an oval, low-density lesion in the tail of the pancreas, and several small nodules in the left lower lung. The RECIST evaluation clearly confirmed progressive disease. A larger dose of sorafenib (600 mg twice daily) was recommended. However, the patient could not tolerate the higher dose owing to uncontrolled severe diarrhea (grade 3), and so in February 2011 sunitinib was carefully selected as a sequential targeted agent.

\section{Sunitinib as second-line targeted therapy}

The patient started sunitinib therapy at a dose of $50 \mathrm{mg} /$ day in February 2011 and experienced mild adverse effects, grade 1 diarrhea. In November 2011, metastases were confirmed in the right kidney, left adrenal gland (Figure 1D), tail of the pancreas, and the liver, and the embolus in the IVC was enlarged. The nodules in both lungs had disappeared. One month later, the metastases in the left adrenal gland and tail of the pancreas had shrunk, but the two metastatic nodules in the liver and the embolus in the IVC were still present. As the comprehensive evaluation at this time was stable disease, the patient continued sunitinib therapy.

In December 2012, the patient was hospitalized for acute urinary retention. Urodynamic tests revealed a maximum voiding flow rate of $1.7 \mathrm{~mL} / \mathrm{s}$ and residual urine of $36 \mathrm{~mL}$ consistent with bladder outlet obstruction induced by $\mathrm{BPH}$. He then underwent a third operation, transurethral resection of the prostate as treatment of the $\mathrm{BPH}$. Postoperative pathological examination confirmed the BPH diagnosis. The patient recovered well and regained normal urination.

By January 2013, the metastasis in the right kidney had increased to $2.7 \times 2.2 \mathrm{~cm}$ (Figure $1 \mathrm{E}$ ), at which time the patient underwent a fourth operation for cryoablation of metastases in both the right kidney and the right hepatic lobe (Figure 1F). Sunitinib therapy was continued until disease progression was confirmed in July 2013.

\section{Everolimus as third-line targeted therapy}

In August 2013, the patient was started on everolimus at a dose of $10 \mathrm{mg} /$ day. He tolerated this treatment well; the only adverse events were grade 2 fatigue and stomatitis. As of 10 February 2014, the patient continues on everolimus therapy with stable disease and ECOG and KPS score of 1 and $90 \%$, respectively. His quality of life, evaluated using the WHOQOL-100 questionnaire, is satisfactory. Close follow-up of the patient is ongoing.

\section{Discussion}

A definitive curative treatment for $\mathrm{mRCC}$ is still lacking. Many factors, such as disease subtype, prognostic factors, comorbidities, and treatment tolerance, may influence the PFS of patients in clinical practice (10). Several novel targeted agents for $\mathrm{mRCC}$ treatment have become available in recent years. Targeted agents such as the vascular endothelial growth factor receptor (VEGFR), tyrosine kinase inhibitors (TKIs) and mammalian target of rapamycin (mTOR) inhibitors have been shown to prolong the PFS of mRCC patients (11). Sorafenib was the first multi-kinase inhibitor (Raf kinases, VEGFRs 1-3, PDGF- $\beta$, Flt-3, c-KIT) to become available (4). Patients who do not respond to immunotherapy may benefit from sorafenib therapy. Sunitinib, a tyrosine kinase inhibitor of VEGFRs, is also effective in the treatment of $\mathrm{mRCC}(6)$. Based on the results of a phase III placebo-controlled study, the mTOR inhibitor everolimus was approved for patients who have failed sunitinib treatment (12). However, resistance to targeted therapy is unavoidable and most patients relapse within 1 year (6). The molecular complexity of the target cells is considered a key factor in the development of resistance to treatment (13).

Adequate management of the drug sequence in the course of treatment is helpful in overcoming drug resistance to targeted agents, and can provide a favorable outcome for $\mathrm{mRCC}$ patients even after progression of the disease (14). Sequential therapy with several targeted agents having different mechanisms of action provides sustainable clinical benefit and should be considered in all patients who can tolerate such treatment (15). Generally, targeted agents are changed according to RECIST criteria, but sometimes the choice of agent is relatively arbitrary in various clinica settings (16). Retrospective studies in Sweden found that first-line treatment with sorafenib was a favorable choice (17), and a Swiss study reported that a sorafenib-sunitinib sequence had a longer combined PFS than a sunitinibsorafenib sequence (18). Based on the positive results of the phase III RECORD-1 trial, current clinical practice guidelines uniformly recommend treatment with the mTOR inhibitor everolimus after initial failure with VEGFR-TKI (19).

This case report describes an $\mathrm{mRCC}$ patient who was treated sequentially with cytokines and targeted therapies including sorafenib, sunitinib, and everolimus. Sequential therapy and cytoreductive surgery provided significant benefit for the patient and contributed to his survival of over 10 years after a diagnosis of advanced RCC. After 38 
months of cytokine therapy, he received 49 months of sorafenib therapy, 30 months of sunitinib therapy, and more than 6 months of everolimus therapy. The treatment intervals for each of the sequentially targeted therapies were significantly longer than those used in previous reports $(18,20)$. The follow-up results also demonstrate the safety of long-term administration of targeted agents.

It is noteworthy that mixed treatment responses, as evaluated by radiological examinations, occurred in the course of combined therapies for this patient. Specifically, new lesions were found in the liver, whereas the nodules in the lung gradually disappeared. This phenomenon appears more frequent with targeted therapy. We propose that the death of cancer cells sensitive to targeted agents might lead to the growth of a resistant population and the spread of resistant progeny to new metastatic sites. Therefore, more definitive criteria to describe this type of change would complement the RECIST criteria as a tool to evaluate treatment response.

Cytoreductive surgeries, including enucleation and cryoablation of tumors, were performed repeatedly in this patient. In our opinion, large kidney tumors are relatively

\section{References}

1. Kim SW, Kim SJ, Park SH, Yang HG, Kang MC, Choi YW, et al. Complete regression of metastatic renal cell carcinoma by multiple injections of engineered mesenchymal stem cells expressing dodecameric TRAIL and HSV-TK. Clin Cancer Res 2013; 19: 415-427, doi: 10.1158/1078-0432.CCR-121568.

2. Danilin S, Sourbier C, Thomas L, Lindner V, Rothhut S, Dormoy $\mathrm{V}$, et al. Role of the RNA-binding protein HuR in human renal cell carcinoma. Carcinogenesis 2010; 31: 10181026, doi: 10.1093/carcin/bgq052.

3. Norian LA, Kresowik TP, Rosevear HM, James BR, Rosean $T R$, Lightfoot AJ, et al. Eradication of metastatic renal cell carcinoma after adenovirus-encoded TNF-related apoptosis-inducing ligand (TRAIL)/CpG immunotherapy. PLoS One 2012; 7: e31085, doi: 10.1371/journal.pone.0031085.

4. Strumberg D. Sorafenib for the treatment of renal cancer. Expert Opin Pharmacother 2012; 13: 407-419, doi: 10.1517/ 14656566.2012.654776.

5. Procopio G, Verzoni E, lacovelli R, Guadalupi V, Gelsomino F, Buzzoni R. Targeted therapies used sequentially in metastatic renal cell cancer: overall results from a large experience. Expert Rev Anticancer Ther 2011; 11: 1631-1640, doi: 10.1586/era.11.154.

6. Wang X, Zhang L, O'Neill A, Bahamon B, Alsop DC, Mier JW, et al. Cox-2 inhibition enhances the activity of sunitinib in human renal cell carcinoma xenografts. Br J Cancer 2013; 108: 319-326, doi: 10.1038/bjc.2012.591.

7. Levy A, Menard J, Albiges L, Loriot Y, Di Palma M, Fizazi K, et al. Second line treatment of metastatic renal cell carcinoma: The Institut Gustave Roussy experience with targeted therapies in 251 consecutive patients. Eur J Cancer 2013; 49: 1898-1904, doi: 10.1016/j.ejca.2013.02.003.

8. Lombardi G, Zustovich F, Donach M, Dalla Palma M, insensitive and respond slowly to medications. Performing appropriate surgical interventions in operable patients could improve their quality of life and result in longer PFS and overall survival.

Multiple therapies including well-planned surgical procedures, prudent decisions in modifying the dosage of targeted agents, and in switching sequential therapy are of great importance in optimizing the clinical management of $\mathrm{mRCC}$. Moreover, close follow-up is indispensable for longterm survival. When disease progression occurs, careful decision-making is essential. In our opinion, individualized therapy should be considered in all mRCC treatment courses. More importantly, with the availability of newer targeted agents such as pazopanib, axitinib, and tivozanib, extensive studies are warranted to determine the optimal choice of sequential therapy and to evaluate new methods for the treatment of $\mathrm{mRCC}$.

\section{Acknowledgments}

Research supported by the Scientific Innovative Project of Shaanxi Province (\#2012KTCL03-03).

Nicoletto O, Pastorelli D. An update on targeted therapy in metastatic renal cell carcinoma. Urol Oncol 2012; 30: 240246, doi: 10.1016/j.urolonc.2009.12.018.

9. Motzer RJ, Agarwal N, Beard C, Bolger GB, Boston B, Carducci MA, et al. NCCN clinical practice guidelines in oncology: kidney cancer. J Natl Compr Canc Netw 2009; 7: 618-630.

10. Escudier B, Szczylik C, Porta C, Gore M. Treatment selection in metastatic renal cell carcinoma: expert consensus. Nat Rev Clin Oncol 2012; 9: 327-337, doi: 10.1038/nrclinonc. 2012.59.

11. Wada $Y$, Takahashi W, Kawano $Y$, Eto M. Current status of pharmacotherapy against metastatic renal cell carcinoma in Japan. Int J Urol 2012; 19: 284-295, doi: 10.1111/j.14422042.2012.02962.x

12. Porta C, Tortora G, Linassier C, Papazisis K, Awada A, Berthold D, et al. Maximising the duration of disease control in metastatic renal cell carcinoma with targeted agents: an expert agreement. Med Oncol 2012; 29: 1896-1907, doi: 10.1007/s12032-011-0016-8.

13. O'Mahony FC, Nanda J, Laird A, Mullen P, Caldwell H, Overton IM, et al. The use of reverse phase protein arrays (RPPA) to explore protein expression variation within individual renal cell cancers. J Vis Exp 2013; pii: 50221, doi: $10.3791 / 50221$.

14. Khattak M, Larkin J. Sequential therapy with targeted agents in metastatic renal cell carcinoma: beyond secondline and overcoming drug resistance. World J Urol 2014; 32 : 19-29, doi: 10.1007/s00345-012-1013-z.

15. Oudard S, Elaidi RT. Sequential therapy with targeted agents in patients with advanced renal cell carcinoma: optimizing patient benefit. Cancer Treat Rev 2012; 38: 981987, doi: 10.1016/j.ctrv.2011.12.009. 
16. Shinohara N. [Renal cell carcinoma-response criteria of molecular targeted therapy and the timing of sequential drugs in patients with advanced renal cell carcinoma]. Gan To Kagaku Ryoho 2012; 39: 1462-1466.

17. Ambring A, Bjorholt I, Lesen E, Stierner U, Oden A. Treatment with sorafenib and sunitinib in renal cell cancer: a Swedish register-based study. Med Oncol 2013; 30: 331, doi: 10.1007/s12032-012-0331-8.

18. Stenner F, Chastonay $\mathrm{R}$, Liewen $\mathrm{H}$, Haile SR, Cathomas $\mathrm{R}$, Rothermundt $\mathrm{C}$, et al. A pooled analysis of sequential therapies with sorafenib and sunitinib in metastatic renal cell carcinoma. Oncology 2012; 82: 333-340, doi: 10.1159/00033
8001.

19. Gonzalez Larriba JL, Espinosa E, Garcia Carbonero I, Garcia-Donas J, Lopez M, Meana A, et al. Sequential therapy in metastatic renal cell carcinoma: pre-clinical and clinical rationale for selecting a second- or subsequent-line therapy with a different mechanism of action. Cancer Metastasis Rev 2012; 31 (Suppl 1): S11-S17, doi: 10.1007/ s10555-012-9354-z.

20. Oudard S. More than 4 years of progression-free survival in a patient with metastatic renal cell carcinoma treated sequentially with sunitinib, everolimus, sorafenib, and temsirolimus. Anticancer Res 2010; 30: 5223-5225. 